\title{
APROXIMACIÓN TEÓRICA E HISTÓRICA PARA EL ANÁLISIS DE LAS POLÍTICAS CIENTÍFICAS DESDE EL ENFOQUE CTS EN AMÉRICA CENTRAL, 1980-2014
}

\section{THEORETICAL AND HISTORICAL APPROACH TO SCIENCE POLICY ANALYSIS FROM THE STS FOCUS ON CENTRAL AMERICA, 1980-2014}

\section{Viviana Guerrero Chacón*}

\begin{abstract}
RESUMEN
El presente artículo pretende aportar a la construcción de un corpus teórico e histórico centroamericano para el análisis de política científica con enfoque cTs, mediante la sistematización de las principales tendencias y posturas teóricas e históricas, principalmente latinoamericanas en la materia. Lo anterior permite no solo determinar la complejidad que conlleva el análisis de política científica sino que visibiliza la necesidad de construir pensamiento endógeno sobre la temática.
\end{abstract}

PALABRAS CLAVE: CENTROAMÉRICA * POLÍTICA CIENTÍFICA * PLANIFICACIÓN DE LA CIENCIA * CIENCIA Y DESARROLLO * INNOVACIÓN CIENTÍFICA

ABSTRACT

This article aims to contribute to the construction of a Central American theoretical and historical corpus for the analysis of scientific policy with STS approach, by systematizing the main trends and theoretical and historical positions, mainly in Latin America in this field. This allows not only to determine the complexity involved in science policy analysis but makes visible the need to build endogenous thinking on the subject.

KEYWORDS: CENTRAL AMERICA * SCIENCE POLICY * SCIENCE PLANNING * SCIENCE AND DEVELOPMENT $*$ SCIENTIFIC INNOVATIONS

* Universidad de Costa Rica.

viviana.guerrerochacon@ucr.ac.cr

$1 \quad$ Este artículo es un resultado parcial del Proyecto de Investigación "Los actores, la percepción y la definición el problema de las políticas científicas en América Central, en el contexto global, y su relación con la cohesión social. 1980-2014. Un análisis histórico desde la perspectiva CTs" (806-B6-191) inscrito en el Programa de Investigación "Ambiente, Ciencia, Tecnología y Sociedad (ACTS) Intersección entre Historia Ambiental y Estudios Sociales de la Ciencia, la Tecnología y la Sociedad (CTS)” (806-B6-901) del Centro de Investigaciones Históricas de América Central de la Universidad de Costa Rica. 


\section{INTRODUCCIÓN}

Mucho se ha escrito sobre la importancia de comprender y analizar los contenidos, contextos y repercusiones relacionados con las políticas científicas, ese grupo de temáticas ha adquirido gran relevancia con el auge de la tecnología y la promulgación de una sociedad del conocimiento, siendo estudiadas en contextos intelectuales diversos, y en latitudes múltiples.

Parece que el impulso a la sociedad del conocimiento ha traído consigo un set de discursos y retóricas que como construcción social, institucional y hasta epistemológica, resultan atrayentes para la academia y la sociedad. Por esta razón, ese conjunto de discursos ha sido analizado e interpretado con parámetros teóricos, políticos o ideológicos disímiles, haciendo que el estudio de la política científica sea diverso y complejo.

Esa diversidad se expresa en una gran cantidad de textos, ofertas interpretativas $y$ posturas analíticas que varían dependiendo la región del mundo en que aparezcan. Europa $y$ Estados Unidos enarbolan estudios pioneros y fundantes de este eje de análisis. Por su parte, Latinoamérica no se queda atrás, pues ha conformado un grupo de textos que ofrecen sesudos análisis sobre la temática.

Pese a la diversidad de posturas, referentes interpretativos $y$ regiones en que se analizan las políticas científicas, salta a la vista que América Central sigue siendo en este y otros temas, una región poco explorada académicamente, lo cual ofrece dos posibilidades que pretenden sugerirse en este artículo: la necesidad de construir teoría que permita analizar la política científica desde una mirada centroamericana y la edificación de un constructo histórico narrado desde nuestra subjetividad, $y$ no desde el imaginario extranjero, principalmente efectuado por agencias de cooperación, como los análisis técnicos del Banco Interamericano de Desarrollo u otros organismos internacionales.

Este no es el primer intento de sistematizar ambas posturas, pues existen varios e importantes esfuerzos de pensar la política científica desde la región, principalmente elaborados desde Costa Rica, pero este texto pretende sistematizar algunas aproximaciones teóricas e históricas, que contribuyan posteriormente a la elaboración de esas dos grandes tareas pendientes: la teorización e historización de las políticas científicas en América Central.

\section{ACERCAMIENTO A UNA TEORIZACIÓN DE POLÍTICAS CIENTÍFICAS PARA AMÉRICA CENTRAL}

Sin duda, el estudio y análisis de política pública es una vieja tradición de estudio en el campo de la Ciencia Política o de la Administración Pública, incluso de la Sociología. El auge de esta rama de pensamiento quizá tenga que ver con la idea de que la política pública es la cara visible del accionar de un Estado; en la política pública se convierten en realidad, las promesas políticas de una campaña electoral o quizá tenga que ver con que la política pública debe dar respuesta a las inquietudes o problemas de la ciudadanía y que debe estar comandada por aquello que es de interés público (Díaz, 2014).

Independientemente de lo que motive esta multiplicidad de interpretaciones, lo cierto es que muchas son las vertientes teóricas con las que se puede efectuar un análisis de política pública. Tradiciones que incluyen desde el análisis por fases o policy cycle de Bellavance (1989), visiones pragmáticas como la de Peter May (1996), visiones procedimentales como la de Hammond, Keeney y Rafia (1999) o desde distintos enfoques teóricos como el corporatismo, el public choice, el welfare economics, la teoría de clase, el estatismo o el neoinstitutcionalismo (Hérnandez, 1999).

También han proliferado visiones críticas que introducen elementos económicos y sociales al análisis, como la visión de Franco y Lanzaro (2006), la de Oszlack y O'Donnell (1984), quienes no solo critican a las propuestas analíticas procedimentales, sino que introducen al análisis, las relaciones de poder o la economía política.

Además de la gran diversidad de enfoques con los que se analiza la política pública, también se encuentra una profesionalización en temáticas específicas, la ciencia social optó por dividir el análisis según ramas, por lo que se han generado herramientas teóricas para 
analizar políticas de salud, financieras, culturales o sociales. En ese marco nace también el afán de construir herramientas propias del análisis de política científica.

En primera instancia, la importancia de analizar la política científica se justifica sobre la base de que esta funciona como uno de los elementos constitutivos de la construcción de un modelo de desarrollo de economía basada en el conocimiento, o más generalmente, como un fundamento primordial de lo que ha sido llamado sociedad del conocimiento, que posee las siguientes características:

1) En el sector económico: el cambio de una economía productora de mercancías a otra productora de servicios; 2) En la distribución ocupacional: la preeminencia de las clases profesionales y técnicas; 3) Como principio axial: la centralidad del crecimiento teórico como fuente de innovación y formulación política de la sociedad; 4) Como orientación futura: el control de la tecnología y de las contribuciones tecnológicas y 5) Para la toma de decisiones: la creación de una nueva "tecnología intelectual” (Bell, 1976, p.30 citado en Marrero, 2007, p.65).

La sociedad del conocimiento no es el único referente sociopolítico que debe ser tomado en cuenta al hacer análisis de política científica, ya que dada la discursividad de los organismos internacionales y de los gobiernos centroamericanos, debe también considerarse la noción de la economía basada en el conocimiento, es decir, "una economía en la cual el conocimiento, es creado, adquirido, transmitido y usado cada vez más efectivamente por empresarios, organizaciones, individuos y comunidades, para alcanzar un mayor nivel de desarrollo económico y social" (González, Vicente-León y Charberlain, 2004, p.8), tendencia que impera en la actualidad en la lógica con la que la mayoría de las veces se hacen las políticas científicas.

Esta noción imperante en el siglo XXI, sugiere que el desarrollo tecnológico es la fuerza fundamental no solo de la economía sino también de la sociedad (ideas que fueron señaladas en la caracterización de la sociedad del conocimiento). Esta noción definida por Joseph Schumpeter, replantea las ideas básicas de la economía capitalista, a saber: el proceso de producción, tal y como la plantea la economía clásica, está basado en componentes materiales como el trabajo, la tierra y el capital. Pero a esa base se le debe añadir una fuerza inmaterial que permita pasar del mero crecimiento económico (crecimiento de la producción) al verdadero desarrollo económico (transformaciones socio-culturales), esa fuerza inmaterial es el efecto del cambio sociotecnológico por medio de la innovación (Montoya, 2004).

El desarrollo o desenvolvimiento económico supone una evolución tecnológica de la sociedad, la cual está sostenida sobre la base de los procesos de innovación. Pero para que exista un verdadero avance, la innovación no debe ser incremental sino radical, entendida como:

a. La introducción de nuevos bienes de consumo en el mercado. b. El surgimiento de un nuevo método de producción y transporte. c. Consecución de la apertura de un nuevo mercado. d. La generación de una nueva fuente de oferta de materias primas. e. Cambio en la organización de cualquier organización o en su proceso de gestión (Montoya, 2004, p.211).

La propuesta no solo de Schumpeter sino de organismos y gobiernos impulsores de este esquema de desarrollo es apuntalar las capacidades del sujeto social promotor de innovación radical: el "empresario innovador"; $y$ el desarrollo de empresas de ese talante: fundamentalmente aquellas de base tecnológica (Montoya, 2004).

El último momento del nuevo proceso productivo, no termina únicamente con la producción innovadora sino que tendrá éxito y será parte del verdadero desarrollo económico en la medida en que sus producciones sean absorbidas por el mercado o por la sociedad mediante educación técnica, telecomunicaciones, salud, entre otros. Aunque se menciona la utilización social de los productos innovadores, tanto en Schumpeter como en otros textos, se evidencia que el sujeto central del proceso es la empresa 
y el empresario innovador (González, VicenteLeón y Charberlain, 2004).

Todo lo anterior debe, según la teoría, ser impulsado a través de las políticas públicas que promuevan principalmente dos elementos: políticas educativas de capacitación de recurso humano útil para la empresa innovadora y el fortalecimiento del Sistema Nacional de Innovación en cada país, cuyo objetivo es otorgar las condiciones sociales, institucionales, entre otros, que faciliten la tarea de la empresa innovadora, fomentando reducciones arancelarias, atracción de inversión, propiedad intelectual, trasferencia de tecnología, ventajas de libre comercio, etc. (González, Vicente-León y Charberlain, 2004, p.25-26). Por lo tanto, el análisis de política científica, debe tomar en cuenta esos aspectos esenciales pero desde una mirada crítica, intentando determinar los intereses subyacentes a ello, las repercusiones sociales y la real efectividad de sus propuestas.

La construcción de la sociedad del conocimiento, de la economía basada en el conocimiento y de la innovación radical sugieren la importancia crucial de la ciencia, la tecnología y la innovación, las cuales han sido ideas hegemónicas a nivel global, sin que la región latinoamericana escape de esto. Pero es por ese impacto discursivo, por la fuerza con que esas ideas se han impulsado y por sus consecuencias sociales, que se debe estudiar su contenido críticamente, desentrañando la opacidad de sus intereses y evidenciando cómo esa hegemonización ha llegado a convertirse en pensamiento único.

Albornoz define el pensamiento único como aquel que:

... reposa sobre tres pilares macroeconómicos ortodoxos: rigor monetario, rigor presupuestario y flexibilidad salarial. En ciencia y tecnología, el pensamiento único se basa en la hegemonía casi absoluta de la óptica de la innovación por sobre cualquier otra dimensión en base a la cual pudiera ser orientada la actividad científica (1997, p.97).

Para la región, el autor aconseja un análisis reposado y crítico frente a esa tendencia tecnócrata internacional, para determinar su pertinencia en el marco de la resolución de necesidades sociales que la región requiere.

Por otra parte, debe tenerse en cuenta que para este artículo se entiende política científica como aquello que "hace referencia al conjunto de políticas que pueden adoptar los estados y en particular los gobiernos en relación con la ciencia" (Albornoz, 2007, p.50) o también "como las 'medidas colectivas' que toma un gobierno para fomentar, de un lado, el desarrollo de la investigación científica y tecnológica $y$, de otro, a fin de utilizar los resultados de esa investigación para objetivos políticos generales" (Salomon, 1977, p.45-46 citado en Elzinga y Jamison, 1996, p.2).

Si bien parece que la política científica es simplemente sumar el concepto de política pública con el de ciencia y tecnología, en realidad el asunto es más complejo que eso, valga aclarar por tanto, tres aspectos sobre esa complejidad. El primero, es la diferencia entre política científica y política de la ciencia:

Esta última se refiere a la interacción entre la ciencia y el poder, esto es: la movilización de la ciencia como un recurso en las relaciones internacionales, la utilización de la ciencia por parte de los grupos de presión o de las clases sociales para aumentar su respectivo poder e influir en la sociedad, y el ejercicio del control social sobre el conocimiento (Elzinga y Jamison, 1996, p.2) [dicha distinción será de suma utilidad en el proceso analítico].

El segundo aspecto, es que el análisis de política científica no puede detenerse solamente en la descripción de aquello que los gobiernos deciden hacer para el desarrollo de una agenda científico-tecnológica, eso es solo el primer paso, ya que se debe trascender hacia un análisis del juego de poder inmerso en la política científica e incluso, determinar la "relación de los hombres de ciencias con las estructuras de poder [...] no sólo como consejeros científicos del gobierno, sino como partícipes en los procesos de toma de decisiones políticas" (Albornoz, s.f, p.11). 
El tercer aspecto es que el análisis debe recurrir a la comprensión de lo que Ruivo (1994) ha llamado el paradigma de la política científica, "este modelo abstracto describe la evolución histórica de la política. En este concepto, la política científica es vista como una política preocupada por el uso y regulación del sistema de investigación” (Ruivo, 1994, p.157). Por ello, el análisis debe contemplar la contextualización, la historización y la noción paradigmática que impera en los contenidos de la toma de decisión.

Hacer análisis de política científica implica desentrañar múltiples dimensiones que se materializan en un plan o una ley, pero que traen de fondo una lucha de intereses, una cultura política y hasta un juego ideológico que debe ser visibilizado en el análisis. Para Elzinga y Jamison (1996), el análisis debe contemplar:

... la mayor y menor importancia que adquiere de forma cíclica el discurso sobre la política científica en un proceso de despolitización y repolitización; los factores sociales y políticos que están detrás de los cambios de las principales direcciones que toma la política científica; la interacción de diversas culturas en relación a la forma de diseñar las políticas, $y$ los problemas de ordenar y explicar las pautas y transformaciones de la política científica (p.3).

En otras palabras, el análisis de política científica debe concentrarse en los procesos sociales, políticos, epistemológicos y hasta ideológicos, los cuales terminan materializándose en un documento de planificación, su implementación y sus repercusiones, a su vez, en los actores que componen el proceso, los recursos que lo sostienen o el contexto en que se enmarca, es decir, descifrar la "cultura de las políticas" que subyace al proceso:

Estas culturas [...] representan intereses sociales y políticos diversos y sus posiciones se inspiran en bases institucionales $y$ tradiciones distintas. Cada cultura tiene su propia percepción de las políticas, incluyendo supuestos doctrinales, preferencias ideológicas e ideales de la ciencia, así como distintas relaciones con quienes ostentan el poder político $y$ económico. Se podría también decir que éstas son los principales componentes de la política científica y tecnológica (Elzinga y Jamison, 1996, p.4).

Aunado a ello, se sugiere la importancia de introducir el análisis de las tendencias internacionales materializadas en la influencia de organismos internacionales en las decisiones de la región:

La mayor parte de esta acción ha sido instrumentada por organismos internacionales de tipo político o financiero - las Naciones Unidas con sus diversos programas y organizaciones ad hoc, la Organización de Estados Americanos y el Banco Interamericano de Desarrollo para el caso particular de América Latina, etc.- - pero también han tenido una participación activa organismos oficiales y privados de las grandes potencias industrializadas, a través de misiones de asistencia técnica, intercambio de investigadores, planes de becas para graduados, etcétera (Herrera, 1995, p.117).

Limitarse a la cuantificación de dicha "ayuda" conllevaría a invisibilizar las implicaciones políticas y sociales, por eso el análisis debe ser sistemático en comprender no solo el componente financiero, las repercusiones y compromisos que los Estados adquieren a cambio de dicha "asistencia técnica", sino también determinar las consecuencias ideológicas de esos ofrecimientos internacionales, para terminar apuntando los efectos sociales de la toma de decisión basada en esa organización internacional de la política científica.

Además, el estudio de la política científica debe incluir un análisis de la comunidad científica como tal, en el marco de una planificación científica que tiene rasgos de tecnocracia, es decir, la racionalidad técnica imperante constantemente hace apelaciones a la preponderancia del papel de las personas científicas en la construcción de política pública: 
Si la racionalidad científica tiende a enseñorearse en el plano de la política económica, con tanta mayor autoridad se ínstala en el de la política científica $y$ tecnológica. No es extraño que, en tal contexto, los científicos tiendan a verse a sí mismos y a la "comunidad" que conforman, como los únicos poseedores de los conocimientos necesarios para orientar el sentido de las políticas públicas en esta materia (Albornoz, 1997, p.98).

Por esta razón es de fundamental importancia comprender su integración, motivaciones e influencia en la configuración de las políticas científicas.

Otro aspecto relevante es la introducción de la variable política al análisis, es decir, los elementos propios:

... de las ciencias políticas, se deberían agregar preguntas tales como: ¿quiénes son los actores que participan y deben participar en la elaboración de la agenda política?, ¿quiénes se benefician del esfuerzo público en materia de ciencia $y$ tecnología?, ¿cómo estimular el esfuerzo de supuestos actores que en la realidad se muestran remisos a adoptar un papel activo en relación con el desarrollo tecnológico?, ¿con qué tipo de incentivos? (Albornoz, 1997, p.106)

Esos elementos pueden dividirse en dos ejes: el análisis de los recursos de poder de los actores que participan de procesos de políticas científicas, en su planificación, ejecución o evaluación, lo cual implica necesariamente recurrir a explicitar los mecanismos autoritarios o democráticos en que se toman decisiones; $y$ un segundo eje que tiene que ver con la participación ciudadana en el proceso.

Como se explicó previamente, las políticas públicas han sido definidas como la forma en que los gobiernos responden a las demandas de la ciudadanía, convirtiendo intenciones en acciones (Bellavance, 1989), lo cual supone no solo que los elementos que componen la política pública derivan necesariamente de lo que es interés público (Díaz, s.f.), sino que dichos componentes deben ser originados en un proceso democrático, deliberativo y participativo, en el cual la ciudadanía manifestó aquello que le preocupa, es parte del consenso por el que se establece cómo resolver esas preocupaciones y solicitar la rendición de cuentas sobre el resultado de ese consenso. En otras palabras:

En un régimen democrático, las políticas públicas constituyen un aspecto sustancial para su consolidación. En primer lugar, implican la acción de gobierno en un entorno plural, de intereses diversos, donde las problemáticas son también plurales $y$ diversas de acuerdo a los intereses $y$ valoraciones de las personas. Requiere construir procesos abiertos y sistemáticos de deliberación para consensuar los problemas que se han de enfrentar [...] Una ciudadanía informada, activa y participativa es un componente distintivo de las políticas públicas en democracia, las cuales deben contemplar mecanismos de participación en condiciones de igualdad para determinar las problemáticas a atender (Arellano y Blanco, 2013, p.8).

Por lo anterior, el análisis de política científica debe comprometerse con ambos aspectos políticos, a saber, la comprensión de los usos y formas de los recursos de poder con que cuentan los actores, así como, el análisis de la democratización y la participación ciudadana de las decisiones que en ella se manifiesta.

Por último - aunque no menos importante- está el análisis de las implicaciones éticas, sociales y ambientales de la política científica, esta vertiente tiene su origen en los Estudios de la Ciencia, la Tecnología y la Sociedad (o enfoque стs), estos:

... tiene[n] por objeto el estudio de la naturaleza social del conocimiento científico-tecnológico y sus incidencias en los diferentes ámbitos económicos, sociales, ambientales y culturales de las sociedades occidentales, primordialmente. A los estudios CTs también se les conocen como estudios sociales de la ciencia 
y la tecnología (Osorio, 2001 citado en Quintero, 2010, p.224).

Este enfoque pretende - desde un punto de vista interdisciplinario e integrando aspectos de la Sociología, la Antropología e incluso la Filosofía- se discuta sobre:

... la dimensión social de la ciencia y la tecnología, tanto desde el punto de vista de sus antecedentes sociales como de sus consecuencias sociales $y$ ambientales, es decir, tanto por lo que atañe a los factores de naturaleza social, política o económica que modulan el cambio científicotecnológico, como por lo que concierne a las repercusiones éticas, ambientales o culturales de ese cambio (García et ál. 2001, p.7)

Asimismo, incluye la tradición europea de cts, en la cual se contextualiza la ciencia $y$ se determina la influencia de los factores sociales en esta; $y$ la tradición estadounidense, en la cual se apela al debate sobre las consecuencias éticas y ambientales de la Ciencia (García et ál. 2001).

Puede deducirse de lo anterior, que el análisis de política científica no es simple metodológica ni interpretativamente, ya que supone el desafío de complejizar el proceso introduciendo todas las variables expuestas:

El análisis de las políticas públicas pone de manifiesto la trama de actores y su articulación en torno al estado. La naturaleza de dicha relación y los intereses prevalecientes quedan así de manifiesto en su dimensión estrictamente política, que incluye aspectos técnicos, sin que éstos disminuyan la politicidad de las decisiones a tomar, ni eliminen la necesidad de elegir y consensuar los fines que se pretendan (Albornoz, 1997, p.105).

El desafío de hacer análisis de política científica integrando todos los elementos anteriormente expuestos, es mayúsculo pero necesario, principalmente en una zona como América Central, que necesita con urgencia independizar sus políticas públicas pero también su análisis académico.

ACERCAMIENTO A LA HISTORIA DE LAS POLÍTICAS CIENTÍFICAS EN AMÉRICA CENTRAL 1980-2014

En cuanto a este segundo elemento, también han sido múltiples los esfuerzos que pretenden cimentar una historización de lo que han sido las políticas científicas en América Latina, algunas de esas intervenciones incluso datan de los años 50, pero rindió frutos principalmente en los 60 y 70 en lo que ha sido denominado "Pensamiento Latinoamericano en Ciencia, Tecnología y Sociedad" (PLACTS):

[Los] análisis de estos exponentes latinoamericanos tuvieron el mérito de señalar el carácter ideológico y político de los enfoques tecnocrático-imitativos, identificaron las limitaciones del quehacer científico-dependiente en América Latina (el "cientificismo") y enfatizaron la necesidad de un "proyecto" que tuviera como norte la eliminación de la pobreza, de las inequidades flagrantes $y$, en general, del subdesarrollo científico, tecnológico y económico de la región (Yarza, 2004, p.196).

Estos grupos cuestionaron la dependencia tecnológica hacia las potencias mundiales, incluso haciendo un llamado a una independencia radical que generara un desarrollo tecnológico endógeno para alivianar la crisis social y económica de la región: "la idea de autodeterminación significa que la solución del subdesarrollo corresponde a los países en desarrollo, que tienen que buscar soluciones creativas, mediante la utilización de sus propios recursos" (Herrera, 1983 citado en Casas, 2004, p.260), es decir que los recorridos sociohistóricos realizados en la región latinoamericana, han partido desde un punto de vista crítico del modelo en ejecución.

Esas críticas fueron dirigidas a que la conformación de la lógica de las políticas científicas, estuvieran comandadas por los organismos internacionales: 
El papel jugado por la Organización de los Estados Americanos (OEA) fue de gran importancia para el diseño de los instrumentos de política científica y tecnológica adoptados por América Latina. En forma convergente, también UNESCO contribuyó al primer diseño institucional de la política científica y tecnológica en la región. Del éxito de aquellos esfuerzos da cuenta el hecho de que los consejos de ciencia y tecnología fueran creados en forma mimética y casi simultánea en muchos países (Albornoz, 2009, p.66).

Este elemento ha sido comprobado en países latinoamericanos, pero en el caso centroamericano, da alcance a un rastreo en documentos e instrumentos de política para su demostración.

En cuanto a la historización propiamente de la política científica en América Central entre 1980 y 2014, pueden hacerse algunas aseveraciones preliminares. La primera de ellas es que, como asegura el PLACTS para otros países, en gran medida la orientación política de América Central durante la época anterior (esencialmente los 60 y 70 ) tuvo un fuerte componente cepalino:

Hacia los años sesenta la ciencia y la tecnología fueron ocupando un lugar cada vez más central en la construcción discursiva del desarrollo, aunque gran parte de los sistemas institucionales de ciencia y tecnología surgidos en este proceso adoptó un patrón mimético alentado externamente por algunos organismos internacionales (Albornoz, 2013, p.113).

Justamente porque la economía se sustentaba en la sustitución de importaciones, es que la ciencia demandaba una transferencia de tecnología extranjera, principalmente para beneficiar el agro (Albornoz, 2009), lo cual evidencia poco esfuerzo para la producción de conocimiento endógeno, tal y como lo criticaba el PLACTS (Casas, 2004).

En los 80, con la entrada en vigencia del modelo neoliberal del Consenso de Washington, el ajuste, la apertura y la privatización también impactaron la forma de hacer ciencia y tecnología, principalmente desestructurando las instituciones estatales que hacían algún tipo conocimiento de científico para darle paso a una conformación meramente administrativa de lo científico:

...el modelo neoliberal, con su lógica aperturista de integración competitiva al mercado internacional, valoró negativamente los esfuerzos anteriores, que fueron señalados como lentos, costosos e ineficientes, por lo que se promovió la asociación con capitales extranjeros para promover una vía privilegiada de fomento de la CyT (Viales 2010, p.28).

Por supuesto, este aspecto no se dio únicamente en la política científica sino en la política pública en general.

Durante la etapa siguiente, se cimientan las bases del ligamen del conocimiento científico y el desarrollo económico, discurso promovido nuevamente por los organismos internacionales, idea que se promocionó en toda la región, no siendo una excepción América Central: "la relación entre conocimiento $y$ desarrollo se plantea ahora como la fórmula básica que deben adoptar los países que desean transitar hacia mejores estadios de crecimiento $y$ desarrollo social" (Casas, 2004, p.265).

En otras palabras, la concepción de la economía basada en el conocimiento promueve una agenda de relación directa entre el conocimiento científico y el desarrollo económico que ha sido aplicada en todo el mundo. Su mecanismo de convencimiento para los países subdesarrollados, ha sido la promesa del combate a la inequidad social a través de la revitalización económica mediante el mejoramiento de la productividad $y$ la competitividad en base al progreso científico, esa percepción ha conducido a Mario Albornoz (1996), a decir que más que de política se ha tratado de una ficción, una "gestión ilusoria" (Yarza, 2004, p.198), esta lógica "venía rodeada, eso sí, de una carga valorativa que la presentaba como el instrumento más apto para solucionar dos de los grandes problemas latinoamericanos: la transformación 
de la estructura productiva y el aumento de la equidad social" (Albornoz, 2013, p.112).

Lo cierto es que ese discurso ha permeado hasta la médula a las políticas científicas en toda la región latinoamericana, transformando los intereses de la ciencia y la tecnología, a la institucionalidad pública e incluso, a las universidades estatales, homogeneizando las tendencias dominantes, lo cual explica que la manifestación de las políticas científicas (con algunas excepciones, por supuesto) sean prácticamente idénticas en la región: agregar el componente innovación a la ciencia y tecnología, creación de fondos de incentivos a la innovación, construcción de sistemas de innovación, escasa inversión privada, adquisición y no producción de tecnología, responsabilidad de ejecución en el sector público, comprender la vinculación universitaria como transferencia de tecnología, tendencia altamente económica y poco social del desarrollo científico y tecnológico, entre otros (Albornoz, 2013).

Esto conlleva una factura social que es indiscutible y que con sus matices se expresa también en toda la región: privatización del conocimiento, asociación de la política científica con la política industrial y no con la social, "el posicionamiento monopólico u oligopólico, las concentraciones de capital y la educación elitista" (Albornoz, 2013, p.122), el mimetismo de países industrializados, agudización de la pobreza y la inequidad, comprensión de la innovación solo como algo tecnológico, poco uso de la ciencia y tecnología para resolver carencias sociales en cuanto a la salud, educación, vivienda o alimentación. Finalmente:

...los países latinoamericanos no solamente deben tomar nota de la importancia del conocimiento científico y tecnológico y de la innovación, sino también de la necesidad de controlar su difusión $y$ aplicación en el contexto de un programa integrador capaz de garantizar la equidad, gestionar los riesgos $y$ fijar un rumbo de desarrollo sostenible (Albornoz, 2013, p.122).

En este sentido, América Central no es lejana a esa realidad regional, la intención fundamental de los gobiernos de los últimos años ha sido:

...fortalecer la infraestructura para la formación de recursos humanos, aunque paulatinamente se incorporaron otras inquietudes vinculadas con la necesidad de articular la producción de conocimientos con el desarrollo de nuevas tecnologías que llevaran a procesos innovadores y al crecimiento de los sectores productivos (Casalet y Buenrostro, 2014, p.166).

Por ende, se ha sido aplicando el modelo propulsado por organismos internacionales, agencias de cooperación y gobiernos recientes, prácticamente en toda la subregión, con diferencias y matices en la forma, tiempo y resultados, pero sin excluir a ningún país.

Pese al éxito con el que se han integrado las políticas científicas, las agendas de economía basada en el conocimiento e impulso a la innovación en América Central, se ha cuestionado la eficiencia y eficacia de la toma de decisiones de los Estados en cuanto a la ciencia y la tecnología, fundamentalmente una crítica a la carencia en la inversión económica necesaria:

Esto se evidencia en el bajo porcentaje de recursos invertidos en relación con el PIB, el que va desde el 0,0023\% en El Salvador (cifra que representa la inversión más baja de la subregión), hasta el $0,41 \%$ en el caso de Costa Rica. Cabe recordar que el porcentaje de inversión en torno al PIB sugerido por la UNESCO para los países en vías de desarrollo corresponde como mínimo a un $1 \%$ (Cooperación Latinoamericana de Redes Avanzadas, 2008, p.364)

Asimismo, la falta de identificación de lineamientos estratégicos del país, falta de recursos humanos especializados y poca vinculación Estado-Universidad-Empresa (Cooperación Latinoamericana de Redes Avanzadas, 2008).

Incluso, en análisis efectuados por la Cooperación Latinoamericana de Redes Avanzadas (2008) y la Organización de Estados 
Americanos, se señala criticando a los países centroamericanos, que aunque los esfuerzos en planificación y creación de institucionalidad se han apegado a las directrices internacionales:

... hay aún una escasa vinculación entre la actividad científica y el sistema económico. Al respecto, llama la atención que si bien es transversal el interés por convocar a participar a la empresa privada en el desarrollo de un sistema científico $y$ tecnológico vigoroso, hay muy pocos instrumentos concretos para asociar la actividad científica con el campo productivo y de incentivos para la innovación empresarial" (REDCLARA, 2008, p.380).

También se destaca una crítica a la falta de liderazgo de los sectores productivos en direccionar las políticas y acciones a sus intereses, por ejemplo, se señala "la ausencia de un rol proactivo del sector productivo industrial de la región, en la propuesta de políticas y acciones específicas de desarrollo científico y tecnológico en pro del incremento de su propia competitividad y capacidad de supervivencia" (Machado, 2004, p.70).

En este sentido, lo que se observa es un cuestionamiento al escaso éxito de llevar a la práctica las nociones del "empresario innovador", no las repercusiones o impactos sociales de tal fracaso, nótese por tanto que la linealidad discursiva en cuanto a la innovación y desarrollo económico es un constructo sólido pero acrítico que va desde 10 internacional a lo nacional.

Frente a esa incapacidad de ejecución del modelo, la región ha iniciado desde 1976 con la creación de la Comisión para el Desarrollo Científico y Tecnológico de Centroamérica y Panamá (CTCAP), y en 1990 con la Declaración de Puntarenas, un proceso de integración que ha pretendido "aumentar los micro- y macroniveles de complejidad de los sistemas productivos $y$ de innovación. Eso supone diseñar instrumentos $y$ programas que estimulen el desarrollo de capacidades y promuevan cambios en los procesos hacia una estructura productiva con mayores complementariedades" (Casalet y Buenrostro, 2014, p. 189), esto demuestra que la región sigue apostando por las estrategias de economía del conocimiento que han sido cuestionadas en otras latitudes.

La subregión ha concentrado esfuerzos, principalmente en la última década en la creación de una institucionalidad fuerte que le permita cumplir con los mandatos internacionales, esa institucionalidad permitió el desarrollo de una capacidad fundamentalmente técnica que ha llevado a la región a un crecimiento relativo, pese a ello, se mantiene la característica de ser una zona dependiente del conocimiento exógeno (CEPAL, 1999).

La ingeniería institucional en ciencia y tecnología creció de mejor manera en Costa Rica y Panamá, pues lograron crear una institución de planificación propia para el sector, mientras que en los demás países lo que existe (aunque también existe en Costa Rica) es un Consejo de Ciencia y Tecnología: "la creación de estos consejos de ciencia y tecnología en los demás países data de fecha más reciente, entre 1992 y 1993, por lo que la articulación y coherencia operativa con la política de desarrollo vigente es todavía objeto recurrente de ajuste" (CEPAL, 1999, p.20).

La existencia de Consejos Científicos en toda la región ha promovido que las políticas científicas tengan amplias semejanzas entre sí, en el entendido que los organismos internacionales influyeron igual en toda la región, es por eso que:

Más allá de esta similitud en las formulaciones generales, son metas compartidas el logro de la productividad y competitividad para mejorar la inserción internacional, así como la necesidad de desarrollar especializaciones productivas de calidad para participar en el mercado mundial (CEPAL, 1999, p.23).

Con lo anterior, se evidencia la solidez de esa influencia no solo en la ingeniería institucional sino también en el contenido de la política.

Finalmente, se puede determinar que la subregión de América Central no es un elemento aislado en la configuración de las tendencias internacionales sobre ciencia y tecnología, lo que resulta interesante es que esa 
configuración ha sido "emergente o incompleta" (Padilla, 2013) respecto a los alcances que se han logrado obtener en otras latitudes, principalmente en Asia.

Pese a ello, América Central sigue siendo complaciente con los modelos exógenos que exigen una vinculación de la política científica $y$ el sector productivo, la competitividad o la economía basada en el conocimiento. Cabe preguntarse si el modelo está cumpliendo sus expectativas sociales en cuanto a la inequidad social, como su nivel de cumplimiento en tareas productivas, técnicas y ambientales en el marco de una región que necesita crecer y solventar problemáticas agudas y complejas.

Por eso, el análisis teórico e histórico de políticas científicas con mirada centroamericana, debe ser crítico respecto a las promesas y realidades en la materia, promesas que pueden ser sintetizadas en el siguiente fragmento, la apuesta por un:

...cambio estructural, caracterizado por un tránsito hacia actividades y sectores más intensivos en conocimientos tecnológicos y un mayor dinamismo de la productividad, permitiría a las economías centroamericanas crecer a mayores tasas, generar empleos mejor remunerados $y$ apropiarse de mayores ganancias como resultado de su participación en cadenas globales de valor (Padilla, 2013, p.51)

Un análisis reflexivo y crítico de esas promesas, es uno de los eslabones que se necesitan para transitar hacia a un modelo de política científica que incluyan las necesidades y requerimientos sociales, éticos y ambientales.

\section{CONCLUSIONES}

El estudio de las políticas científicas resulta en sí mismo una tarea compleja que requiere un esfuerzo de análisis diacrónico y sincrónico para alcanzar a comprender más allá de los instrumentos, el sentido y la orientación de sus intereses. Por eso, enriquecer el análisis con posturas teóricas sólidas y de construcción latinoamericana permite una mayor apropiación de ese fenómeno político.
Con aportaciones teóricas de la Sociología, la Filosofía y la Antropología se puede cimentar un constructo teórico autóctono centroamericano que permita estudiar-nos con una mirada más cercana.

Esta sistematización pretende hacer un recorrido por otras latitudes tanto teóricas como históricas para tomar aquello que es útil, con el fin de aportar a un marco teórico endógeno para el análisis de política científica. Ese recorrido puede sintetizarse en dos alcances: el primero es teórico y consiste en la necesidad de profundizar en el estudio de política científica implica incluir las tradiciones de análisis de esta, reuniendo las posturas de análisis por fases (que sin duda permiten, aunque sea con fines reflexivos, segmentar en momentos una política) con posturas críticas que aportan una visión de conjunto.

Además de ello, el análisis a profundidad también recopila y comprende las decisiones gubernamentales como tal, las agendas de ciencia y tecnología, las culturas y paradigmas de las políticas, el componente internacional, el aporte, influencia e intereses de la comunidad científica, los recursos de poder de los actores involucrados, la participación ciudadana y la toma de decisiones democrática, así como, las implicaciones sociales, éticas y ambientales de la política. La integralidad de estos elementos aportará en la construcción de un análisis complejizado de la ciencia y la tecnología.

El segundo alcance es de tipo histórico, ya que el recorrido por etapas y tendencias permite ver-nos como parte de un constructo mayor, como parte de una tendencia internacional que no es casual, que es intencionada y que tiene consecuencias. Por esta razón, el análisis de política científica debe incluir una contextualización histórica regional, para evitar el desapego de lo particular (los instrumentos de política) respecto a lo general (el contexto socio-histórico).

Integrar ambos alcances en el análisis de política científica contribuirá a un valioso estudio en niveles de profundidad, permitiendo una mejor comprensión de los significados, símbolos y repercusiones de esa toma de decisión. La necesidad de seguir dando pasos en esa vía está abierta. 
BIBLIOGRAFÍA

LIBROS

Arellano, D. y Blanco, F. (2013). Políticas Públicas y Democracia. México: Instituto Federal Electoral. Acceso en agosto de 2015. http:// www.ine.mx/docs/IFE-v2/DECEYEC/ EducacionCivica/CuadernosDivulgacion/ CuadernosDivulgacion-pdfs/CUAD_30_ definitivo.pdf

Bellavance, M. (1989). Las Políticas Gubernamentales (elaboración, gestión y evaluación). En: Arias, F. Traducción de Las Politicas Gubernamentales (elaboración, gestión y evaluación). Heredia: Universidad Nacional.

Franco, R. y Lanzaro, J. (2006). Política y políticas públicas en los procesos de reforma de América Latina. México, México: Facultad Latinoamericana de Ciencias Sociales.

Herrera, F. (2009). Inconsistencia e incertidumbre. Las políticas de ciencia, tecnología e innovación en Costa Rica. En: Viales, R., Amador, J. y Solano, F. (2009). Concepciones y Representaciones de la Naturaleza y la Ciencia en América Latina. San José, Costa Rica: Universidad de Costa Rica.

Herrera, F. y Cuevas, F. (2010). Actores, intereses $y$ percepciones de la comunidad político-científica en torno a la formulación del problema de las políticas de ciencia, tecnología e innovación en Costa Rica en la primera década del Siglo xxI. En: Viales, R. (Ed.). El contexto, los problemas y los actores de la definición de políticas científicas para la cohesión social en América Latina: Una visión desde Costa Rica. San José: Sociedad Editora Alquimia 2000.

Herrera, F. y Gutiérrez, J. (2011) Conocimiento, Innovación y Desarrollo. San José, Costa Rica: Impresión Gráfica del Este. Acceso en agosto de 2015. catedrainnovacion. ucr.ac.cr/librocid.pdf

May, P. (1996). Claves para diseñar opciones de políticas. En: Aguilar, L. Problemas públicos y agenda de gobierno, estudio introductorio y edición. Segunda edición. México, México: Miguel Ángel Porrúa.

Oszlak, O. y O'Donnell, G. (1984). Estado y Politicas Estatales en América Latina: hacia una estrategia de investigación. En: Nef, J. y Flores, G. edit. Administración Pública, perspectivas críticas. San José, Costa Rica: Instituto Centroamericano de Administración Pública.

Padilla, R. (2013). Sistemas de innovación en Centroamérica. Fortalecimiento a través de la integración regional. Comisión Económica para América Latina y el Caribe (CEPAL), Chile. Acceso en setiembre de 2015. http://www.cepal. org/es/publicaciones/2622-sistemasde-innovacion-en-centroamericafortalecimiento-traves-de-la-integracion

Raiffa, H. et al. (1999). Decisiones inteligentes. Guía práctica para tomar las mejores decisiones. Bogotá, Colombia: Grupo Editorial Norma.

Viales, R. (Ed.), (2009). Concepciones y representaciones de la naturaleza y la ciencia en América Latina. San José: Editorial de la Universidad de Costa Rica.

Viales, R. (Ed.). (2010) El contexto, los problemas y los actores de la definición de politicas científicas para la cohesión social en América Latina: Una visión desde Costa Rica. San José: Sociedad Editora Alquimia 2000.

Viales, R. (Ed.). (2010). El contexto, los problemas y los actores de la definición de politicas científicas para la cohesión social en América Latina: Una visión desde Costa Rica. San José: Sociedad Editora Alquimia 2000.

Viales, R., Amador, J. y Solano, F. (2009). Concepciones y Representaciones de la Naturaleza y la Ciencia en América Latina. San José: Universidad de Costa Rica.

\section{PUBLICACIONES PERIÓDICAS}

Albornoz, M. (1997). La política científica y tecnológica en América Latina frente al desafío del pensamiento único. REDES, IV, (10), 95-115. 
Albornoz, M. (2007). Los problemas de la ciencia y el poder. Revista cts, 3, (8) 47-65.

Albornoz, M. (2009). Desarrollo y políticas públicas en ciencia y tecnología en américa latina. RIPS. Revista de Investigaciones Politicas y Sociológicas, 8, (1), 65-75.

Albornoz, M. (2013). Innovación, equidad y desarrollo latinoamericano. ISEGORÍA. Revista de Filosofía Moral y Política, (48), 111-126, ISSN: 1130-2097.

Casalet, M. y Buenrostro, E. (2014). La integración regional centroamericana en ciencia, tecnología e innovación: un nuevo desafío. Economía: Teoría y práctica, (40), 165-193.

Casas, R. (2004). Conocimiento, tecnología y desarrollo en América Latina. Revista Mexicana de Sociología, 66 (Especial), 255-277.

Dagnino, R., Thomas, H. y Davyt, A. (1996). El pensamiento en ciencia, tecnología y sociedad en América Latina: una interpretación de su trayectoria. En: Revista REDES, 3, (7), 13-51.

Elzinga, A. y Jamison, A. (1996). El cambio de las agendas políticas en ciencia y tecnología. En: Revista Zona Abierta (75/76), 91-132.

Estébanez, M.E. (2004). Conocimiento científico y políticas públicas: un análisis de la utilidad social de las investigaciones científicas en el campo social. Espacio Abierto, Cuaderno Venezolano de Sociología, 13, (1), 7-37.

Hernández, G. (1999). El análisis de las Políticas Públicas: una disciplina incipiente en Colombia. En: Revista de Ciencias Sociales, Universidad de los Andes, (4), 80-91.

Hernández, M. (2011). Análisis Comparativo del Discurso de las Políticas Públicas de Ciencia, Tecnología e Innovación En Colombia. Hologramatica - Facultad de Ciencias Sociales, 2, (14),137-167.

Herrera, A. (1995). Los determinantes sociales de la política científica en América Latina. Política científica explícita y política científica implícita. Redes, 2 , (5),117-131.

Marrero, A. (2007). La sociedad del conocimiento: Una revisión teórica de un modelo de desarrollo posible para América Latina. Arxius de Ciencias Sociales, (17), 36-73.

Montoya, O. (2004). Schumpeter, innovación y determinismo tecnológico. Scientia et Technica, 2, (25), 209-213.

Oszlak, O. (2014) Políticas Públicas y Capacidades Estatales. Revista Forjando, Banco de la Provincia de Buenos Aires, (5).

Oszlak, O. (2007). Políticas Públicas, Democracia y Participación Ciudadana. Voces del Sur, (4), 8-13.

Quintero, C. (2010). Enfoque Ciencia, Tecnología y Sociedad (стs): perspectivas educativas para Colombia. Revista del Instituto de Estudios en Educación Universidad del Norte, (12), 222239. Acceso en junio de 2015. http:// rcientificas.uninorte.edu.co/index.php/ zona/article/download/1151/719

Ruivo, B. (1994). Phrases or Paradigms of science policy? Science and Public Policy, 21, (3), 157-164.

Vanegas, L. (2009). La relación ciencia, tecnología y desarrollo en las políticas públicas del gobierno de Nicaragua. Revista Ciencias Económicas, 27, (2), 107-122.

Viales, R., Arellano, A. y Granados, R. (2012). Perceptions about the political-scientific community and its role in formulating the problems of public policy for science, technology and innovation in Costa Rica. Science and Public Policy, (39), 613-617.

Viales, R. y Clare, P. (2006). El Estado, lo transnacional y la construcción de comunidades científicas en la Costa Rica liberal (1870-1930). La construcción de un "régimen de cientificidad". Diálogos Revista Electrónica de Historia, 7, (2), 145-168.

Yarza, C. (2004). Sobre los usos de Schumpeter en el discurso de la política científica. Revista cts, 1, (2), 195-209. 


\section{TEXTOS ELECTRÓNICOS}

Albornoz, M. (s.f). Política Científica. Módulo de contenido para el dictado del curso.

Acceso en setiembre de 2015. http://www. oei.es/ctsiima/albornoz.pdf

Comisión Económica para América Latina y el Caribe-CEPAL. (1999). Tecnología y pobreza en el istmo centroamericano. Acceso en agosto de 2015. http://www. cepal.org/es/publicaciones/3223tecnologia-y-pobreza-en-el-istmocentroamericano

Cooperación Latinoamericana de Redes Avanzadas. (2008). Politicas, instituciones e instrumentos para el desarrollo de la ciencia, la tecnología y la innovación. Capítulo 4. Análisis de Tendencias. Acceso en junio de 2015. http://docplayer.es/8543508-4-analisisde-tendencias.html

Crespi, G. (2010). Nota Técnica sobre el Sistema Nacional de innovación de Costa Rica. San José, Costa Rica: Banco Interamericano de Desarrollo.

Díaz, L. (2014). Nuevos Temas en el Análisis de Políticas Públicas. Acceso en junio de 2015. https://campus.usal.es/ dpublico/ areacp/materiales/6.3.nuevasperspectiv as.pdf

García, E. et ál (2001). Ciencia, Tecnología y Sociedad: una aproximación conceptual. Cuadernos de Iberoamérica, Organización de Estados Iberoamericanos para la Educación, la Ciencia y la Cultura. Acceso en agosto de 2015. www. oei.es/ctsiparaguay/cp4elec.pdf

Lederman, D. et ál. (2014). El emprendimiento en América Latina, Muchas empresas y poca innovación. Washington, Estados Unidos: Banco Internacional de Reconstrucción y Fomento/
Banco Mundial. Acceso en noviembre de 2015. http://www.worldbank.org/ content/dam/Worldbank/document/ LAC/EmprendimientoAmericaLatina_ resumen.pdf

Machado, F. (2004). Globalización y políticas de ciencia y tecnología en América Central. Temas de Iberoamérica: Globalización, Ciencia y Tecnología. Acceso en agosto de 2015. www.oei.es/ salactsi/machado.pdf

Maggi, C., Rivas, G. y Sierra P. (2012). Fortalecimiento del Sistema de Ciencia, Tecnología e Innovación de Costa Rica. San José, Costa Rica: Banco Interamericano de Desarrollo. Acceso en junio de 2015. http://www.iadb.org/ en/publications/publication-detail,7101. html?id=45786

González, R., Vicente, A. y Chamberlain, J. (2004). I\&D, innovación y transferencia de tecnología en el sector productivo costarricense más orientado hacia la economía basada en el conocimiento. San José, Costa Rica: Banco Interamericano de Desarrollo. Acceso en noviembre de 2015. http://www.caatec. org/CAATEC/publicaciones/otros/ Innovacion.pdf

Organización para la Cooperación y Desarrollo Económico, (2012). La Estrategia de Innovación de la OCDE, Empezar Hoy el Mañana. ocDE-Foro Consultivo Científico y Tecnológico. Acceso en setiembre de 2015. http://www.foroconsultivo.org.mx/ libros_editados/estrategia_innovacion_ ocde.pdf

Fecha de ingreso: 11/07/ 2016

Fecha de aprobación: 18/08/2016 